\title{
Lidil
}

Revue de linguistique et de didactique des langues

Acquisition et enseignement de la morphographie

\section{Le processus relationnel}

Écrire à plusieurs voix

Henry Tyne

\section{CpenEdition}

\section{Journals}

Édition électronique

URL : http://journals.openedition.org/lidil/1023

DOI : $10.4000 /$ /idil. 1023

ISSN : 1960-6052

Éditeur

UGA Éditions/Université Grenoble Alpes

\section{Édition imprimée}

Date de publication : 1 décembre 2004

Pagination : 188-190

ISBN : 2-914176-11-2

ISSN : $1146-6480$

Référence électronique

Henry Tyne, "Le processus relationnel », Lidil [En ligne], 30 | 2004, mis en ligne le 29 janvier 2008 , consulté le 22 septembre 2020. URL : http://journals.openedition.org/lidil/1023 ; DOI : https://doi.org/ 10.4000/lidil.1023 
sitera bien d'autres ouvrages de ce type. À moins que le travail des chercheurs n'ait aucune prise sur les décisions politiques...

Cyril Trimaille

BILLIEZ J. (1997): Bilinguisme, variations, immigration: regards sociolinguistiques, Dossier présenté en vue de l'Habilitation à diriger des recherches, 2 volumes, Université Stendhal-Grenoble 3.

LeONETTI-TABOADA I. (1991): "Stratégies identitaires et minorités », Migrants Formation, n 86, p. 54-73.

De Gaulmyn, M.-M., R. Bouchard \& A. Rabatel (dir.), 2001, Le processus rédactionnel: Écrire à plusieurs voixx, Paris, L'Harmattan, 317 p. ISBN : 2-2475-0883-8.

Cet ouvrage collectif, qui réunit plus d'une vingtaine d'auteurs $^{8}$, traite des interactions dans et par le processus rédactionnel, que ce soit au niveau de la genèse des textes écrits, captée au vif, ou au niveau du méta-texte après coup. Par des voies d'approche variées, les différentes contributions participent chacune à sa manière à une réflexion autour de la rencontre entre oral et écrit, longtemps tenus à distance dans la logique du «grand partage».

Dans l'introduction (p.9-16), les coordinateurs (M.-M. de Gaulmyn, R. Bouchard et A. Rabatel) expliquent le contexte scientifique et intellectuel dans lequel s'insère l'ouvrage, qui prend sa source dans une rencontre interdisciplinaire entre chercheurs en Sciences Humaines (voir aussi de Gaulmyn,

8. Par ordre alphabétique: J. Andriessen, D. Apotheloz, J.-P. Bernié, A.-C. Berthoud, J.-M. Besse, N. Bonnardel, R. Bouchard, C. Brassac, A. Camps, A. Chevalier, P. Coirier, J. David, L. Gajo, M.-M. de Gaulmyn, O. Guasch, J. Miecznikowski-Fünfschilling, M. Milian, L. Mondada, A. Moulin, A. Piolat, S. Plane, A. Rabatel, T. Ribas, J. Vacherand-Revel. 
p.31-48). S'ensuit alors une présentation des différentes contributions. Celles-ci sont réunies en deux parties: l'une, intitulée «La rédaction conversationnelle: variations sur un même corpus» (p. 17-168), rassemble des observations effectuées sur un même extrait de corpus; l'autre, intitulée «Processus rédactionnels, cognition et apprentissage» (p.169310 ), est consacrée à diverses approches de la description des rédactions de textes en interaction.

Les différentes contributions se donnent pour objectif de poursuivre trois thèmes centraux, censés faire le lien entre les trois composantes des Sciences Humaines représentées ici (linguistique, didactique, psychologie). Ces trois thèmes sont: la dynamique de la production collaborative d'écriture; la problématique des unités oralo-graphiques de la langue; l'importance du contexte dans la description et l'interprétation des activités cognitives d'écriture en collaboration.

Dans la première partie, après une présentation de la recherche lyonnaise sur la rédaction conversationnelle (de Gaulmyn, p. 31-48), plusieurs chercheurs sont invités à interpréter un extrait de corpus mettant en scène deux étudiants étrangers de niveau avancé (Paulo et Méité) qui accomplissent ensemble un devoir par écrit (discussion plus acte rédactionnel). De cette première partie, certains aspects récurrents transparaissent dans les différentes analyses (dont certaines auraient mérité de s'appuyer sur des données plus vastes) telles l'émergence des formes de l'écrit (par quels processus ?), la négociation individuelle des places et des stratégies, la différence interpersonnelle. Par exemple, pour Apotheloz (p.49-66), les deux individus n'ont pas la même façon de procéder dans la production du texte: Paulo parait plus «nominaliste » que Méité, qui opte plutôt pour une syntaxe phrastique. Selon cet auteur, Méité finit par se rallier aux choix de Paulo. Or, A. Rabatel (p. 67-88) suggère que Méité pèse plus sur les choix de rédaction et qu'elle "refuse même d'envisager les formulations de Paulo» (p. 74). Plus loin, S. Plane (p.103128) remarque néanmoins que c'est l'obstination de Paulo qui impose les règles de l'écrit (par exemple l'adoption du pluriel, qui sera suivie par Méité): «Dans le couple que forment les deux co-scripteurs, Paulo apparait davantage comme un garant de la règle [...] Il prend également en charge l'initia- 
tive de la formulation. Méité, quant à elle, manifeste plus d'inventivité, en créant une anecdote et en injectant le mot 'travail' qui va permettre d'enrichir la représentation des devoirs» (p. 127). Plus loin encore, R. Bouchard (p. 129-146) suggère que Paulo est plus sensible à la norme et au genre de production (dissertation scolaire) tandis que Méité parait plus sensible aux acquis extrascolaires.

La deuxième partie, qui présente des recherches non apparentées, met en évidence une pluralité d'approches (pour la plupart des études qualitatives) de la description des rédactions de texte dans l'interaction. Ici, l'écriture est d'autant plus montrée comme une activité sociale située que ces différentes approches traitent de diverses situations véritables d'interaction: rédaction coopérative en milieu industriel, pratiques d'écriture dans le monde académique, débat critique autour d'un article en ligne, etc. Ici il apparait que l'écrit - le fait de rédiger, même - structure le savoir (MiecznikowskiFünfschilling et Mondada, p. 195-220) et organise la représentation du monde (Brassac, p. 171-194). Les dernières pages de l'ouvrage sont consacrées aux résumés des articles.

Si l'entreprise d'un tel ouvrage est louable - surtout à l'heure actuelle où l'on encourage l'interdisciplinarité - le produit final n'est sans doute pas le meilleur reflet de la qualité du travail effectué par ce groupe de chercheurs. Outre les sérieux problèmes de présentation (notamment dans les bibliographies), l'ouvrage manque parfois d'explications et de précisions: les conventions de transcription (p. 22-29) ne font pas mention de l'utilisation de caractères gras et italiques, tout comme elles n'expliquent pas le choix de coder les apprenants Paulo et Méité en $\mathrm{H}$ et $\mathrm{F}$ (le professeur est codé P). Le lecteur attendra la page 130 pour connaitre la nationalité de Paulo et Méité...

Malgré ces problèmes d'édition, l'ouvrage représente toutefois une contribution globalement intéressante, originale, sur la rencontre entre oral et écrit. 